九州大学学術情報リポジトリ

Kyushu University Institutional Repository

\title{
Which Cicadellid Species (Homoptera) are Difficult to Find in a Heterogeneous Habitat?
}

Kamitani, Satoshi

Entomological Laboratory, Faculty of Agriculture, Kyushu University

Saito, Aya

Entomological Laboratory, Faculty of Agriculture, Kyushu University

https://doi.org/10.5109/2651

出版情報: ESAKIA. 41，pp.27-36，2001-03-31. Entomological Laboratory，Faculty of Agriculture， Kyushu University

バージョン :

権利関係 : 
ESAKIA, (41): 27 - 36. March 31, 2001

\title{
Which Cicadellid Species (Homoptera) are Difficult to Find in a Heterogeneous Habitat ?*
}

\author{
Satoshi Kamitani and Aya Saito \\ Entomological Laboratory, Faculty of Agriculture, \\ Kyushu University, Fukuoka, 812-8581 Japan
}

\begin{abstract}
The factors that determine which cicadellid species are easy or difficult to find in a heterogeneous habitat are discussed. The probability of finding a cicadellid and the estimated true species richness were computed by the randomized species accumulation curve. The mean observed richness and estimated true species richness were 15.1 and 27.0 species, respectively. Incidence, temporal abundance, annual abundance, and host plant type were used for the analyses. The most influential factor was the incidence. Temporal abundance also strongly influenced the probability. High incidence and high abundance species were easily detected. A protocol for estimating the gap species is suggested.

Key words: biodiversity, true species richness, incidence, abundance, host plant type, gap species, Cicadellidae.
\end{abstract}

\section{Introduction}

Species richness is one of the most important traits of species diversity, because the number of species occurring at a site is a quantitative measure of biodiversity and allows comparison with other sites (Harper and Hawksworth, 1996). However, a noncorrected value for observed data is usually used to indicate species richness. True species richness is very difficult to assess in the field. Therefore, Colwell and Coddington (1996) proposed a statistical method to estimate true species richness based on the species-by-sample incidence matrix. In their study, the term "sample" refers to a list of species and their abundance at a given site on a given date, and the term "investigation" refers to a matrix of all samples at a site on a given date. Therefore, an investigation consits of some samples.

To estimate the true species richness, species richness was computed by the randomized species accumulation curve to estimate observed species richness, which is the total number of species observed in all samples pooled. In this method, the probability that a species will occur in a given accumulation level depends on the incidence of the species. Therefore, the difficulty or ease of detecting a species in an investigation is closely related to its incidence, and the higher the incidence of the species, the easier it is to detect. However, very few low-incidence species could be found using

* Contribution from Entomological Laboratory, Faculty of Agriculture, Kyushu University, Fukuoka (Ser. 5, No. 65). 
this estimation. Thus, we discuss here the factors related to species that are generally difficult to find in a heterogeneous habitat.

To clarify the factors that affect the probability of encountering a species, we estimate a new component, "gap species", defined as the true species richness minus the observed species richness. The gap species are particularly difficult to find in a survey. To discuss the Alfa-biodiversity, we must know the complete species list collected at a site, as well as the species number. Therefore, it is necessary to obtain a concrete estimate of the gap species. Although a necessary component, no method to determine real species has been proposed, as yet. To obtain this value, it is important to clarify which species are difficult and which species are easy to find. Yamamoto (1998) suggested that butterflies with the following features are difficult to find using a route census method: those that occur during a short seasonal period, are active during a short temporal period in the day, are not of large body size, and have no conspicuous wings. Ishii (1997) pointed out that butterflies that are difficult to find have the following features: they fly very high, usually live near the crown of a tree, occur within a very small area, and remain still. Kamitani and Urano (2000) suggested that rare or transient auchenorrhynchan species are difficult to find in a homogeneous habitat. In this study, we propose a method to estimate the gap species.

We used Cicadellidae to survey Alfa- and beta-biodiversity in this study. This leafhopper family is a very large sap-sucking taxon in Insecta and includes more than 20,000 species in various habitats of the world (Dolling, 1991), thus it should be useful for understanding biodiversity.

\section{Materials and Methods}

\section{Field study}

The local species diversity of Cicadellidae was surveyed at three sites, Motooka, Ino, and Konomi, which are located near Fukuoka City, Japan (Fig. 1). These areas are about $10 \mathrm{~km}$ distant from each other, and are largely covered by secondary evergreen forests. Quercus glauca Thunb. ex. Murray was the dominant tree species in the forests. Samplings were performed along a trail and census length was $1.0 \mathrm{~km}$ in Motooka, 0.6 $\mathrm{km}$ in Ino, and $1.2 \mathrm{~km}$ in Konomi. Investigations were performed 18 times in total, once a month from April to December (2000: 8 May, 15 June, 6 July, 10 August, 8 November, and 27 December in Motooka; 1 June, 7 July, 4 August, 20 September, and 18 October in Ino; 2 April, 3 May, 4 June, 2 July, 1 August, 10 September, and 13 October in Konomi. The cicadellid leafhoppers were collected by a sweeping method with a constant sampling effort, 5 - 10 sweeps per weed colony or tree.

\section{Estimation of species richness}

In order to make use of and analyze the data of sufficiently surveyed investigations, we computed the randomized species accumulation curve for each investigation by EstimateS 6 (Colwell, 2000). With the 300 fold randomized sample order, the following 2 mean values were computed: standard deviation of Sobs and accumulative number of species for each value of the sample number pooled. When the standard deviation of 


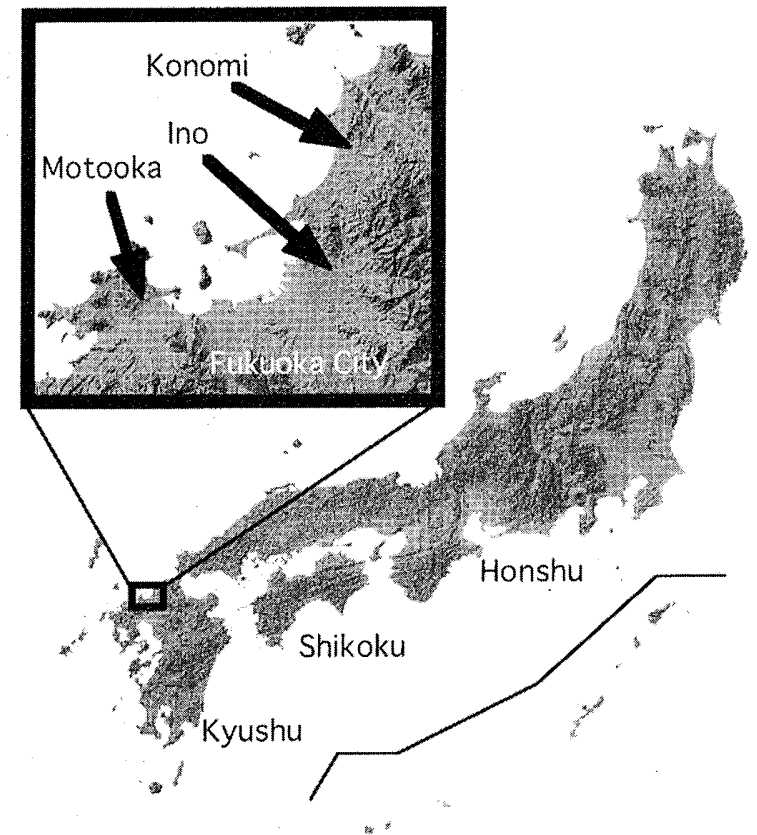

Fig. 1. Study site.

penultimate Sobs reached 0 , the species richness was regarded as sufficiently surveyed and the curves were considered to have reached equalization.

The estimated true species richness was calculated with ICE (Colwell and Coddington, 1994). ICE is a coverage estimator based on incidence, and it is a modification of Chao's estimator.

\section{Relationships}

The investigation rate, which is the proportion of a given Sobs to the final Sobs, was compared for the following 4 factors: incidence, temporal abundance, annual abundance, and host plant type. These factors may influence the difficulty of finding some species in investigations. The incidence, which is the total number of samplings and varies from 1 to the entire sample number, was divided into 5 categories: unique, duplicate, 3 - 5, 6 10 , and $>10$ samples in an investigation. Uniques are species with only one sample in an investigation, and duplicates are those with two samples. The temporal abundance was divided into 4 categories based on the relative proportion to the total number of individuals; $<1 \%,<5 \%,<10 \%$, and $\geqq 10 \%$ of the total. The annual abundance is the total number of investigations in which some adults were collected, and was divided into 4 categories: $1,2,3-5$, and $\geqq 6$ investigations in a year. The host plant type was divided into 3 categories: tree, weed, and tree/weed type. 


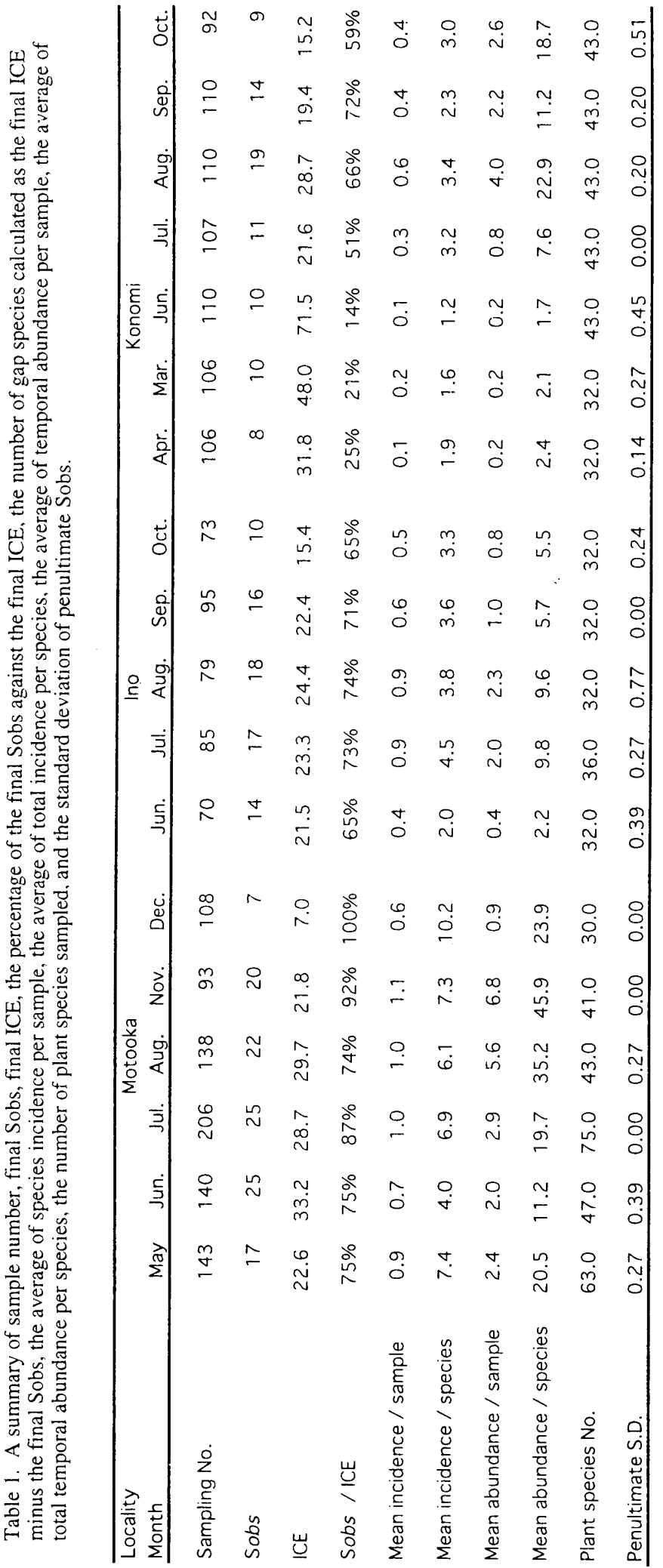



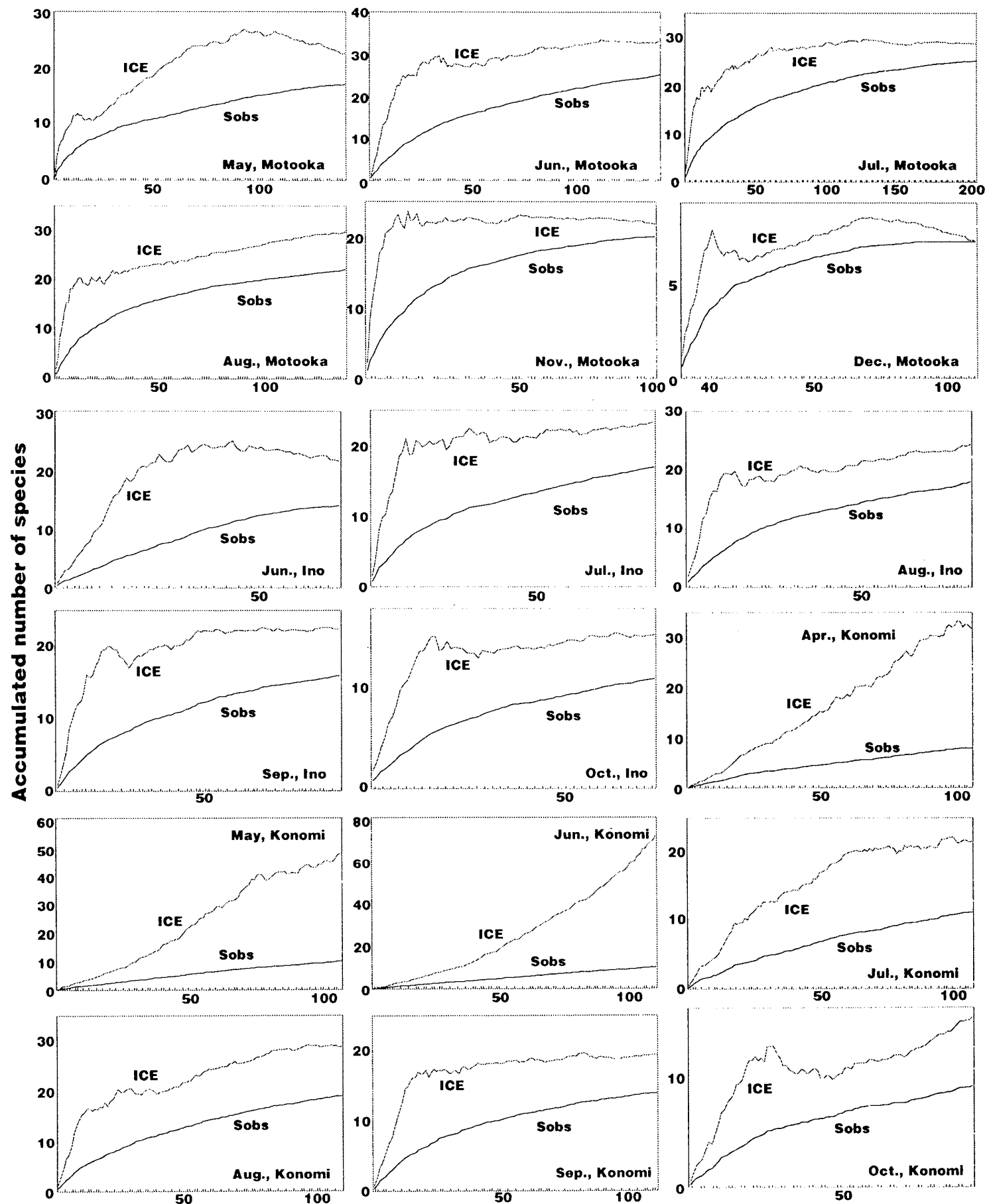

Number of samples pooled

Fig. 2. Accumulative Sobs and ICE curves. 


\section{Results}

\section{Field study}

Table 1 shows a summary of sample number, final Sobs, final ICE, the percentage of final Sobs over final ICE, the number of gap species calculated as the final ICE minus the final Sobs, average species incidence per sample, average total incidence per species, average temporal abundance per sample, average total temporal abundance per species, the number of plant species sampled and the standard deviation of penultimate Sobs. Thirty-nine cicadellid species were collected. The mean observed species richness and estimated true species richness were 15.1 and 27.0 species per investigation, respectively, and the estimated investigation rate was $56 \%$. Throughout the investigations, the average species incidence per sample was 0.6 species, average total incidence per species was 4.6 samples, average temporal abundance per sample was 2.2 individuals, and average total temporal abundance per species was 16.2 individuals.

\section{Estimation of species richness}

All the accumulation curves that are based on Sobs and ICE are shown in Fig. 2. Only 5 of 18 curves reached equalization: July, November, and December in Motooka, September in Ino, and July in Konomi. The average percentage of final Sobs against final ICE was $64 \%$. The mean number of gap species was 11.9 species per investigation.

\section{Components of the cicadellid community}

Uniques and duplicates occupied a high percentage in most investigations and the total data (Fig. 3A). The relative frequencies of the temporal abundance varied among investigations (Fig. 3B). Species for which temporal abundance was less than 5\% constantly occupied a high percentage, but species for which temporal abundance was less than 1\% ranged between 13\% (Motooka, December) and 58\% (Motooka, September). The frequencies of species which appeared in more than 6 or more investigations were very high in all investigations, ranging between 58\% (Motooka, September) and 91\% (Konomi, July) (Fig. 3C). The frequencies of species collected in only 1 or 2 investigations were very low. The frequency of species which utilize trees as host plant was high except for one investigation (Motooka, November) (Fig. 3D).

\section{Species that were difficult to find}

The 4 graphs (Fig. 4) indicate the relationships between each of 4 factors and the investigation level. An investigation level was calculated as a given Sob/the final Sob at a sample accumulation level. The following 5 investigation levels were shown: $50 \%$, $60 \%, 70 \%, 80 \%$, and $90 \%$. In the relationship between the investigation level and incidence, the frequency of uniques was higher than that of other incidences at most of the investigation levels (Fig. 4A). The frequency of uniques and duplicates increased from $60 \%$ to $90 \%$ investigation level. In the relationships between investigation level and temporal abundance, the frequency of $<1 \%$ abundance was the highest at all investigation levels (Fig. 4B). Only $<1 \%$ abundance constantly increased with the level of investigation. In the relationship between investigation level and annual abundance, 


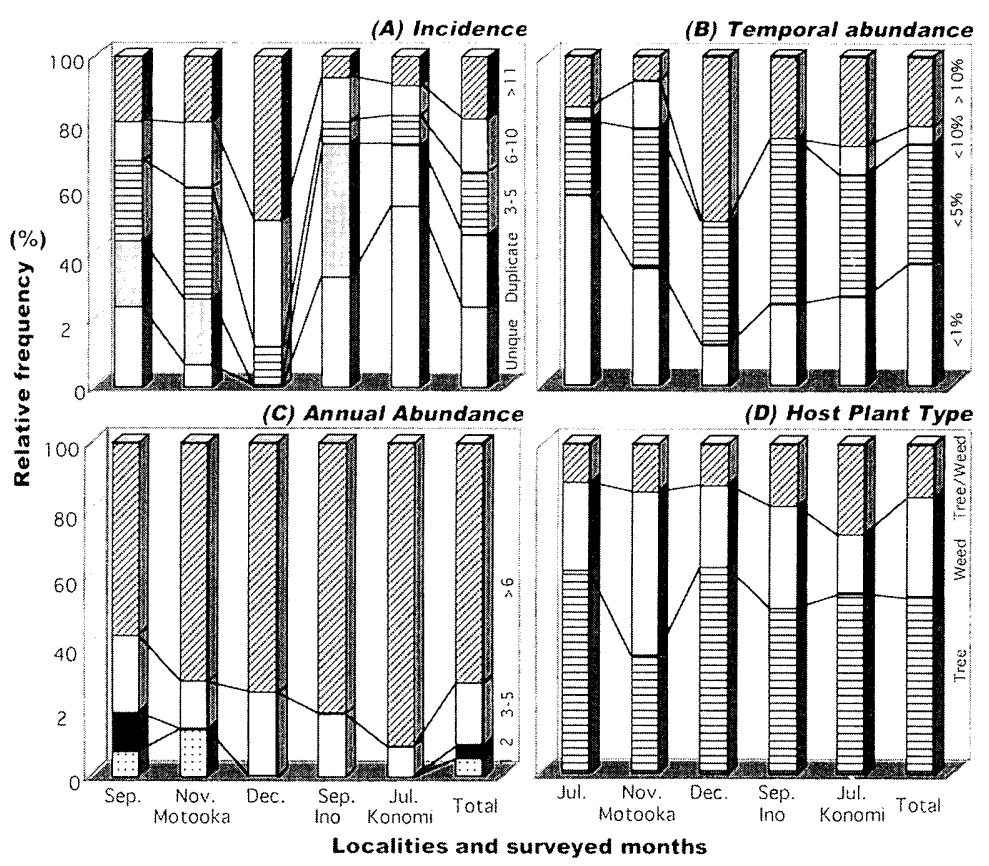

Fig. 3. Relative frequency of species number based on the four factors (A-D).

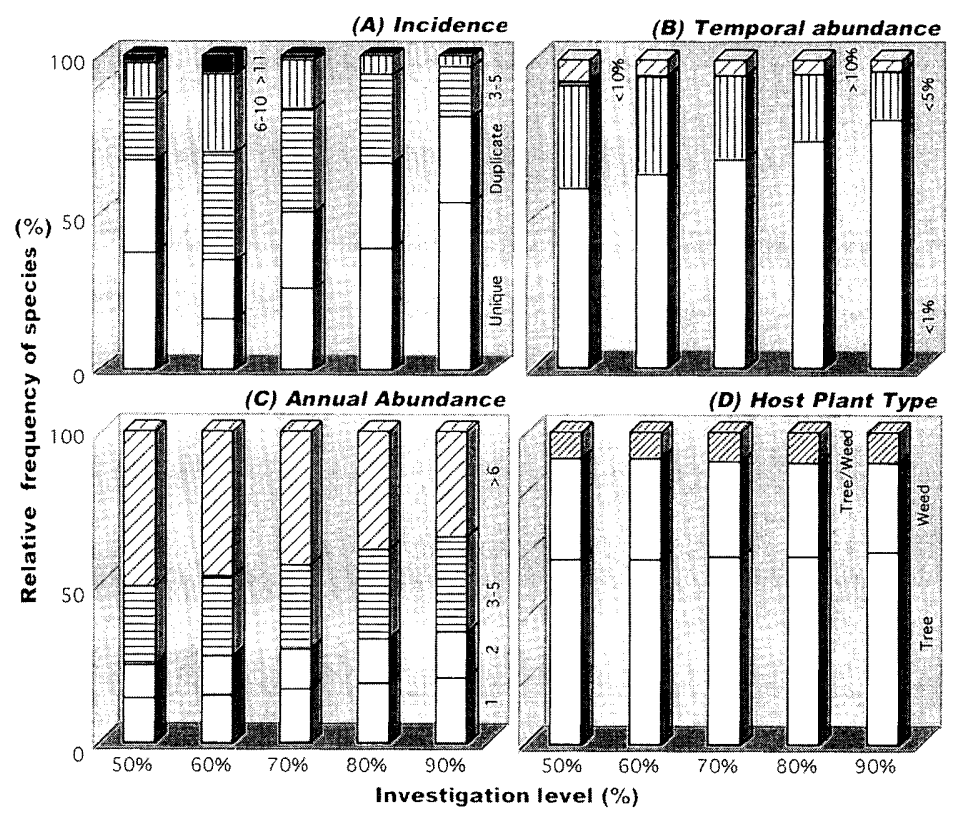

Fig. 4. Relative frequency of species number based on categories of four factors (A-D). 

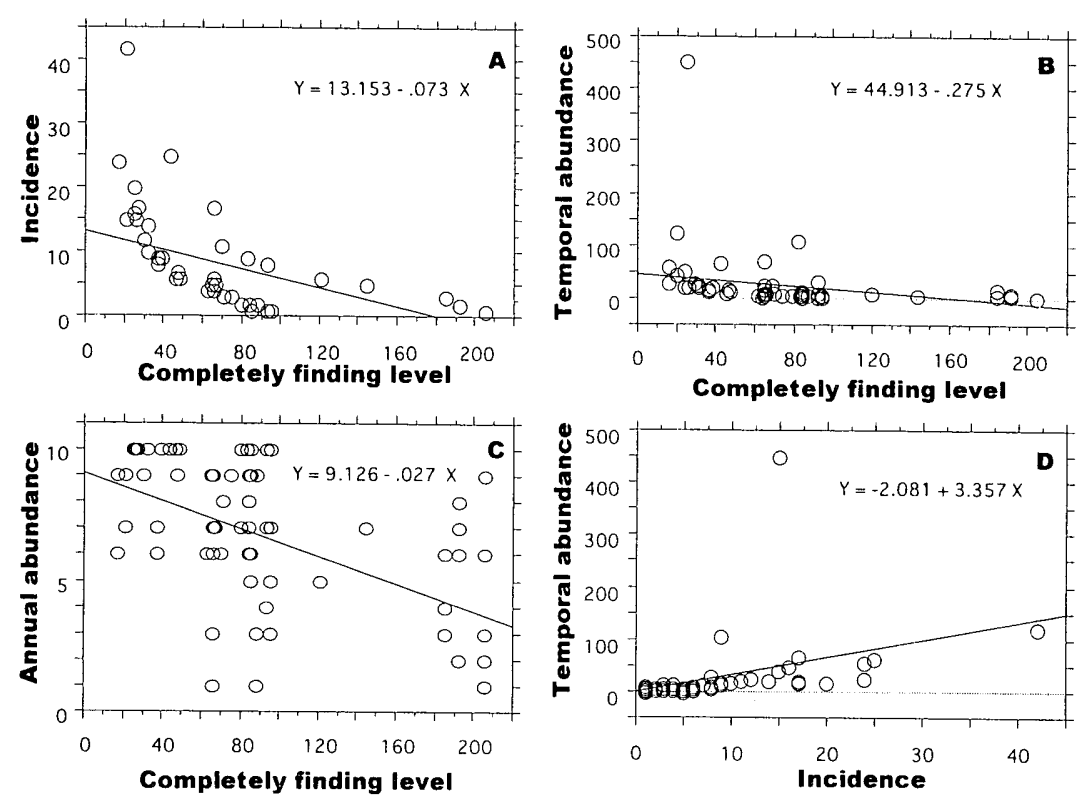

Fig. 5. Correlation among the completely finding sampling number and three factors (A-C9. Correlation between the incidence and temporal abundance (D).

Table. 2. Correlation among the completely finding sampling number and three factors, and between them.

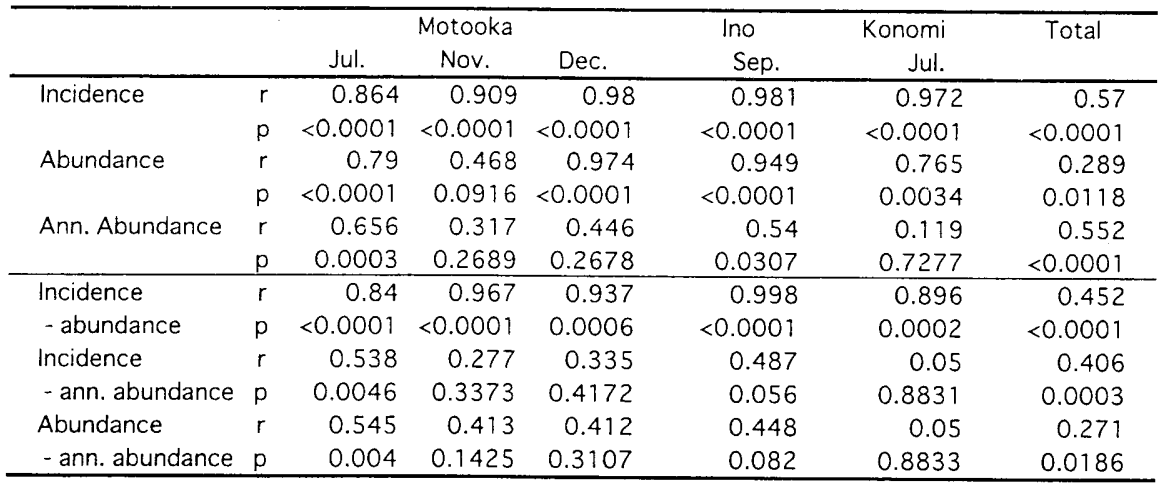

the frequency of $>6$ investigations was always the highest (Fig. 4C). The other 3 categories constantly increased with the level of investigation. In the relationship between investigation level and host plant type, tree type frequency was the highest (Fig. 4D). Only tree type frequency constantly increased with the level of investigation.

Incidence based on total data correlated closely to the completely finding level $(\mathrm{p}<0.0001$, Fig. 5A). A completely finding level was a sample accumulation level at which the probability to find a given species is $100 \%$ in the accumulation curve simulated with the incidence of the species. The correlation coefficient values between sampling number and incidence were all over 0.8 and were strongly significant $(\mathrm{p}<0.0001$, Table 2). The temporal abundance based on the total data also correlated closely to the 
sampling number $(\mathrm{p}<0.01$, Fig. $5 \mathrm{~B})$. Some correlation coefficient values were below 0.8 , but the r-values were strongly significant except for one investigation (Motooka, November). This discrepancy was caused by the high abundance and low incidence of Limassolla multipunctata (Matsumura). The annual abundance based on the total data showed a strong correlation to the sampling number ( $p<0.0001$, Fig. $5 \mathrm{C}$ ), however, with the exception of one investigation (Ino, September), the correlation coefficient values were not significant.

\section{Discussion}

\section{Species that are difficult to find}

The uniques and duplicates were more abundant than others at all investigation levels, and species with higher incidence were more esay to find. In contrast, species with lower incidences were more difficult to find. This tendency is reasonable, because, as mentioned above, the probability of finding a species depends on the incidence in the randomized estimation. Although the probability does not directly depend on temporal abundance, the low abundance species constantly increased with the level of investigation. The annual abundance is not appropriate for discussing probability, because the r-values were not significant. For host plant type, the frequencies at each investigation level did not differ notably. Therefore, incidence and temporal abundance can be hypothesized as the main factors affecting the probability of detecting a species.

\section{How can we estimate gap species?}

Incidence was more significantly correlated with the total number of samples found than abundance (Table 2). If we hypothesize that incidence is the main factor affecting the probability of detection, most gap species will be estimated as high incidence species. Some low incidence species may also be estimated as gap species. In such cases, which low incidence species become gap species? Some of the low incidence species were very abundant, such as L. multipunctata on November, Motooka. Therefore, the high abundance in effect renders the species with low incidence gap species. Abundance is strongly correlated with incidence $(r=0.452, p<0.0001$, Table 2, Fig. 5), and the regression line is:

$$
\mathrm{Y}=-2.081+3.357 \mathrm{X}
$$

where $\mathrm{X}$ represents incidence, and $\mathrm{Y}$ represents abundance. For low incidences of less than 6 samples in an investigation, the correlation was strongly significant $(r=0.442$, $\mathrm{p}<0.01$ ). Most gap species should be estimated as high incidence species. Low incidence and high temporal abundance species should also be estimated as gap species.

To estimate or infer the gap species precisely, we suggest the following protocol: 1) create a model species list, which is a record of all species collected throughout the study site; 2) transform the temporal abundance into incidence by the regression lines between them; 3) arrange the species list in order of incidence or temporal abundance using the higher value of them; 4) exclude species observed in the investigation from the arranged list; 5) select as many higher rank species as the estimated number of gap species. The chosen species should be the gap species. 
The present study was based on these 4 factors: incidence, temporal abundance, annual abundance and host plant type. Detailed studies with other factors are required for further understanding of Alfa- and beta-biodiversity.

\section{Acknowledgments}

We wish to express our sincere gratitude to Prof. J. Yukawa and Assoc. Prof. O. Tadauchi (Entomological Laboratory, Kyushu University) for their guidance. Many thanks are due to Dr. Lisa Filippi (Saga City) for her critical reading of this manuscript. Our cordial thanks are due to Mr. A. Matsunaga, Mr. T. Katsuda, and Miss. M. Nohara for their valuable assistance with the surveys.

\section{References}

Colwell, R. K., 2000. Estimates: Statistical estimation of species richness and shared species from samples. Version 6. User's Guide and application. http://viceroy. eeb.uconn.edu/estimates.

Colwell, R. K. \& J. A. Coddington, 1994. Estimating terrestrial biodiversity through extrapolation. Philosoph. Trans. Roy. Soc. (Ser. B), 345: 101-118.

Colwell, R. K. \& J. Coddington, 1996. Estimating terrestrial biodiversity through extrapolation. pp. 101-140, In Hawksworth, D. L. (ed.) Biodiversity: Measurement and Estimation. Chapman \& Hall, London.

Dolling, W. R., 1991. The Hemiptera. Oxford University Press, London, 274 pp.

Harper, J. L. \& D. L. Hawksworth, 1996. Preface. pp. 5-12. In Hawksworth, D. L. (ed.). Biodiversity: Measurement and estimation. Chapman \& Hall, London.

Ishii, M., 1993. [Transect Counts of Butterflies]. pp. 91-101, In Ishii, M. (ed.) [Decline and Conservation of Butterflies in Japan, II]. Lepidopterol. Soc. Jap., Osaka. (In Japanese.)

Kamitani, S. \& S. Urano, 2000. Species diversity and optimum sampling size of Auchenorrhyncha (Homoptera) in a homogeneous habitat. Esakia (40): 87-94.

Yamamoto, M., 1998. [Route-census method]. pp. 29-43, In Imai, C. \& M. Ishii (eds.) [A Manual to Research Butterflies]. Jap. Soc. Envir. Ent. Zool., Osaka. (In Japanese.) 